\title{
AFFINE HYPERSURFACES WITH PARALLEL CUBIC FORM
}

\author{
FRANKI DILLEN ${ }^{1)}$, LUC VRANCKEN ${ }^{2)}$, \\ AND SAHNUR YAPRAK ${ }^{3)}$
}

\section{§1. Introduction}

As is well known, there exists a canonical transversal vector field on a nondegenerate affine hypersurface $M$. This vector field is called the affine normal. The second fundamental form associated to this affine normal is called the affine metric. If $M$ is locally strongly convex, then this affine metric is a Riemannian metric. And also, using the affine normal and the Gauss formula one can introduce an affine connection $\nabla$ on $M$ which is called the induced affine connection. Thus there are in general two different connections on $M$ : one is the induced connection $\nabla$ and the other is the Levi Civita connection $\hat{\nabla}$ of the affine metric $h$. The difference tensor $K$ is defined by $K(X, Y)=K_{X} Y=\nabla_{X} Y-\hat{\nabla}_{X} Y$. The cubic form $C$ is defined by $C=\nabla h$ and is related to the difference tensor by

$$
h\left(K_{X} Y, Z\right)=-\frac{1}{2} C(X, Y, Z) .
$$

The classical Berwald theorem states that $C$ vanishes identically on $M$, implying that the two connections coincide, if and only if $M$ is an open part of a nondegenerate quadric.

In this paper we will consider the condition $\hat{\nabla} C=0$ for a 4-dimensional locally strongly convex affine hypersurface in $\mathbf{R}^{5}$. Clearly $\hat{\nabla} C=0$ if and only if $\hat{\nabla} K=0$. For surfaces this condition has been studied by M. Magid and K. Nomizu in [MN], where they proved the following;

THEOREM A [MN]. Let $M^{2}$ be an affine surface in $\mathbf{R}^{3}$ with $\hat{\nabla} C=0$. Then either $M$ is an open part of a nondegenerate quadric (i.e. $C=0$ ) or $M$ is affine equivalent to an open part of the following surfaces:

Received October 26, 1992.

1,2) Senior Research Assistant of the National Fund for Scientific Research (Belgium)

3) Research Assistant of Ankara University (Turkey) 
(1) $x y z=1$,

(2) $x\left(y^{2}+z^{2}\right)=1$,

(3) $z=x y+\frac{1}{3} y^{3}$ (the Cayley surface).

A generalization of this theorem to 3 -dimensional locally strongly convex hypersurfaces in $\mathbf{R}^{4}$ is given by the first two authors in [DV1]. There the following classification theorem is proved.

THEOREM B [DV1]. Let $M$ be a 3-dimensional affine locally strongly convex hypersurface in $\mathbf{R}^{4}$ with $\hat{\nabla} C=0$. Then either $M$ is a part of a locally strongly convex quadric (i.e. $C=0$ ) or $M$ is affine equivalent to an open part of one of the following two hypersurfaces:

(1) $x y z w=1$,

(2) $\left(y^{2}-z^{2}-w^{2}\right)^{3} x^{2}=1$.

Comparing Theorem A and Theorem B with the classification of locally strongly convex homogeneous hyperspheres in $\mathbf{R}^{3}$ and $\mathbf{R}^{4}$ in [NS] and [DV3] (homogeneous in the sense used therein), we find that a locally strongly convex affine hypersphere in $\mathbf{R}^{3}$ or $\mathbf{R}^{4}$ is homogeneous if and only if it satisfies $\hat{\nabla} C=0$. In [DV2] it is proved that the hypersurface in $\mathbf{R}^{5}$ with equation

$$
\left(z-\frac{1}{2} x^{2} / u-\frac{1}{2} y^{2} / v\right)^{4} u^{3} v^{3}=1,
$$

is a homogeneous hyperbolic affine hypersphere in $\mathbf{R}^{5}$. It however does not satisfy $\hat{\nabla} C=0$. In the present paper we give a classification of all locally strongly convex affine hypersurfaces in $\mathbf{R}^{5}$ with $\hat{\nabla} C=0$. In particular, our main result is the following theorem.

THEOREM 1. Let $M$ be a 4-dimensional locally strongly convex affine hypersurface in $\mathbf{R}^{5}$ with $\hat{\nabla} C=0$. Then either $M$ is an open part of a locally strongly convex quadric (i.e. $C=0$ ) or $M$ is affine equivalent to an open part of one of the following three hypersurfaces:

(1) $x y z w t=1$,

(2) $\left(y^{2}-z^{2}-w^{2}-t^{2}\right)^{2} x=1$,

(3) $\left(z^{2}-w^{2}-t^{2}\right)^{3}(x y)^{2}=1$.

All examples occurring in the previous theorems are special cases of the following class of hypersurfaces of $\mathbf{R}^{n+1}$ satisfying $\hat{\nabla} C=0$ with equation 


$$
\prod_{i=1}^{k}\left(x_{i ; p_{i}+1}^{2}-\sum_{j=1}^{p_{i}} x_{i, j}^{2}\right)^{p_{i}+1}\left(y_{1} \cdots y_{q+1}\right)^{2}=1
$$

where $n=\sum_{i=1}^{k}\left(p_{i}+1\right)+q$ and

$$
\left(x_{1 ; 1}, \ldots, x_{1 ; p_{1}+1}, x_{2 ; 1}, \ldots, x_{2 ; p_{2}+1}, \ldots, x_{k ; 1}, \ldots, x_{k ; p_{k}+1}, y_{1}, \ldots, y_{q+1}\right)
$$

are affine coordinates of $\mathbf{R}^{n+1}$. The theorems mentioned above show that this class gives all examples of locally strongly convex hypersurfaces with $\hat{\nabla} C=0$ for $n=$ $2,3,4$. This is however not true for $n=5$, as follows from the discussions in [DV2].

All examples occurring are also homogeneous. This property remains true in all dimensions. We will prove this in the final section.

THEOREM 2. Let $M$ be a nondegenerate affine hypersurface in $\mathbf{R}^{n+1}$ with $\hat{\nabla} C=0$. Then $M$ is a locally homogeneous affine sphere.

We will use the formalism and the notations of [N]. For a short survey of the preliminaries that we need in this paper, we refer to [DV1, §2].

\section{§2. The construction of an orthonormal basis}

In this section, we consider an $n$-dimensional, locally strongly convex affine hypersurface $M$ in $\mathbf{R}^{n+1}$ which has parallel cubic form, i.e. which satisfies $\hat{\nabla} C=0$. From [BNS], if follows that $M$ is an affine sphere, so the affine shape operator is $S=\lambda I$.

Since $\hat{\nabla} C=0$ implies that $h(C, C)$ is constant, there are two cases. First if $h(C, C)=0$, then $C=0, h$ being definite, and therefore $M$ is an open part of a quadric. Otherwise, $C$ never vanishes, and we assume this for the remainder of this section.

Let $p \in M$. We now choose an orthonormal basis with respect to the affine metric $h$ at the point $p$ in the following way, similar as in [DV1]. Let $U M_{p}=\{u \in$ $T_{p} M \mid h(u, u)=1$. Since $M$ is locally strongly convex, $U M_{p}$ is compact. We define a function $f$ on $U M_{p}$ by $f(u)=h\left(K_{u} u, u\right)$. Let $e_{1}$ be an element of $U M_{p}$ at which the function $f$ attains an absolute maximum. If $f\left(e_{1}\right)=0$, then $f$ is identically zero, and therefore, $K$ being symmetric, $K=0$. This contradicts our assumption, so $f\left(e_{1}\right)>0$.

Let $u \in U M_{p}$ such that $h\left(u, e_{1}\right)=0$, and let $g$ be a function, defined by $g(t)=f\left(\cos (t) e_{1}+\sin (t) u\right)$. Since $g$ attains an absolute maximum at $t=0$, we 
have $g^{\prime}(0)=0$, which means that $h\left(K_{e_{1}} e_{1}, u\right)=0$. So $e_{1}$ is an eigenvector of $K_{e_{1}}$, say with eigenvalue $\lambda_{1}$. Let $e_{2}, e_{3}, \ldots, e_{n}$ be orthonormal vectors, orthogonal to $e_{1}$, which are the remaining eigenvectors of $K_{e_{1}}$ with respective eigenvalues $\lambda_{2}, \lambda_{3}, \ldots$, $\lambda_{n}$. Further, since $e_{1}$ is an absolute maximum of $f$, we know that $g^{\prime \prime}(0) \leq 0$ and if $g^{\prime \prime}(0)=0$, then also $g^{\prime \prime \prime}(0)=0$. This implies that

$$
\lambda_{1}-2 \lambda_{i} \geqq 0
$$

and

$$
\text { if } \lambda_{1}=2 \lambda_{i} \text {, then } h\left(K_{e_{i}} e_{i}, e_{i}\right)=0
$$

for $i \in\{2,3, \ldots, n\}$. From the apolarity condition we have

$$
\lambda_{1}+\lambda_{2}+\cdots+\lambda_{n}=0 .
$$

Now $\hat{\nabla} K=0$ implies that $\hat{R} \cdot K=0$, and as in the proof of [DV1, Lemma 3.3], this implies that

$$
\left(\lambda_{1}-2 \lambda_{i}\right)\left(-\lambda-\lambda_{i}^{2}+\lambda_{1} \lambda_{i}\right)=0 .
$$

If $\lambda_{1}=2 \lambda_{i}$ for all $i \in\{2,3, \ldots, n\}$, then (2.3) implies that $\lambda_{1}=0$ which is a contradiction. Therefore there is a number $k, 1 \leq k<n$ such that, after rearranging the ordering,

$$
\lambda_{2}=\lambda_{3}=\cdots=\lambda_{k}=\frac{1}{2} \lambda_{1} \text { and } \lambda_{k+1}<\frac{1}{2} \lambda_{1}, \ldots, \lambda_{n}<\frac{1}{2} \lambda_{1} .
$$

Moreover, if $i>k$, then (2.4) implies that

$$
-\lambda-\lambda_{i}^{2}+\lambda_{1} \lambda_{i}=0
$$

Subtracting (2.4) for $i, j>k$, we obtain

$$
\left(\lambda_{i}-\lambda_{j}\right)\left(\lambda_{1}-\left(\lambda_{i}+\lambda_{j}\right)\right)=0 .
$$

But for $i, j>k$ one can check that $\lambda_{1}-\left(\lambda_{i}+\lambda_{j}\right) \neq 0$. Thus $\lambda_{i}=\lambda_{j}$ for $k<i, j$ $\leq n$. Setting $\lambda_{k+1}=\cdots=\lambda_{n}=\mu$ and using (2.3) and (2.5), we have

$$
\begin{aligned}
\mu & =-\frac{k+1}{2(n-k)} \lambda_{1} \\
-\lambda & =\lambda_{1}^{2} \frac{\left((k+1)^{2}+2(k+1)(n-k)\right)}{4(n-k)^{2}} .
\end{aligned}
$$

Therefore we have proved the following result. 
Proposition 2.1. If $M$ is a locally strongly convex hypersurface of $\mathbf{R}^{n}$ with $\hat{\nabla} C=0$, then $M$ is a hyperbolic affine sphere.

\section{§3. Hypersurfaces in $\mathbf{R}^{5}$}

From now on $M$ will be a hypersurface in $\mathbf{R}^{5}$. Then, using the notation of $\S 2$, we have the following cases.

Case $A: k=1$. Then $\lambda_{1}=\frac{3}{2} \sqrt{-\lambda}, \lambda_{2}=\lambda_{3}=\lambda_{4}=-\frac{\sqrt{-\lambda}}{2}$.

So in this case $K_{e_{i}}$ has a 3-dimensional eigenspace corresponding to the eigenvalue $\mu$. Define the function $f_{1}$ to be the restriction of $f$ to this eigenspace and choose $e_{2}$ as the maximum of $f_{1}$, thus $h\left(K_{e_{2}} e_{2}, u\right)=0$ where $u \in U M_{p}$ is orthogonal to both $e_{1}$ and $e_{2}$. Let the function $f_{2}$ be the restriction of $f$ to $B$ where $B=$ $\left\{u \in U M_{p} \mid h\left(u, e_{1}\right)=h\left(u, e_{2}\right)=0\right\}$. We can choose $e_{3}$ as an absolute maximum of $f_{2}$. Then $h\left(K_{e_{3}} e_{3}, u\right)=0$ for $u \in U M_{p}$ with $h\left(u, e_{1}\right)=h\left(u, e_{2}\right)=h\left(u, e_{3}\right)=0$. Finally we can adjust the sign of $e_{4}$ such that $h\left(K_{e_{2}} e_{3}, e_{4}\right) \geq 0$. Resuming, the difference tensor $K$ takes the following form:

$$
\begin{aligned}
& K_{e_{1}} e_{1}=\frac{3 \sqrt{-\lambda}}{2} e_{1}, K_{e_{2}} e_{2}=-\frac{\sqrt{-\lambda}}{2} e_{1}+a e_{2}, \\
& K_{e_{3}} e_{3}=-\frac{\sqrt{-\lambda}}{2} e_{1}+b e_{2}+d e_{3}, K_{e_{4}} e_{4}=-\frac{\sqrt{-\lambda}}{2} e_{1}-(a+b) e_{2}-d e_{3}, \\
& K_{e_{1}} e_{2}=\frac{-\sqrt{-\lambda}}{2} e_{2}, K_{e_{1}} e_{3}=\frac{-\sqrt{-\lambda}}{2} e_{3}, K_{e_{1}} e_{4}=\frac{-\sqrt{-\lambda}}{2} e_{4} \\
& K_{e_{2}} e_{3}=b e_{3}+c e_{4}, K_{e_{2}} e_{4}=c e_{3}-(a+b) e_{4}, K_{e 3} e_{4}=c e_{2}-d e_{4},
\end{aligned}
$$

where $a, b, c, d$ are real numbers and by assumption $a \geq 0, c \geq 0, d \geq 0$. Note that if $a=0$, then the function $f_{2}$ is identically zero, so also $b=c=d=0$.

Case $B: k=2$. Then $\lambda_{1}=4 \sqrt{\frac{-\lambda}{21}}, \lambda_{2}=2 \sqrt{\frac{-\lambda}{21}}$ and $\lambda_{3}=\lambda_{4}=-3 \sqrt{\frac{-\lambda}{21}}$.

Here, we can choose $e_{3}$ in the direction of $K_{e_{2}} e_{2}$, such that $h\left(K_{e_{2}} e_{2}, e_{4}\right)=0$ and $h\left(K_{e_{2}} e_{2}, e_{3}\right) \geq 0$. Also, because of (2.2) we know that $h\left(K_{e_{2}} e_{2}, e_{2}\right)=0$. Here the difference tensor takes the following form:

$$
K_{e_{1}} e_{1}=4 \sqrt{\frac{-\lambda}{21}} e_{1}, K_{e_{2}} e_{2}=2 \sqrt{\frac{-\lambda}{21}} e_{1}+a e_{3},
$$




$$
\begin{aligned}
& K_{e_{3}} e_{3}=-3 \sqrt{\frac{-\lambda}{21}} e_{1}+b e_{2}+d e_{3}+f e_{4}, \\
& K_{e_{4}} e_{4}=-3 \sqrt{\frac{-\lambda}{21}} e_{1}-b e_{2}-(a+d) e_{3}-f e_{4}, \\
& K_{e_{1}} e_{2}=2 \sqrt{\frac{-\lambda}{21}} e_{2}, K_{e_{1}} e_{3}=-3 \sqrt{\frac{-\lambda}{21}} e_{3}, K_{e_{1}} e_{4}=-3 \sqrt{\frac{-\lambda}{21}} e_{4}, \\
& K_{e_{2}} e_{3}=a e_{2}+b e_{3}+c e_{4}, K_{e_{2}} e_{4}=c e_{3}-b e_{4}, K_{e_{3}} e_{4}=c e_{2}+f e_{3}-(a+d) e_{4}
\end{aligned}
$$

where $a, b, c, d, e, f$ are real numbers and by assumption $a \geq 0$.

Case $C: k=3$. In this case, we have $\lambda_{1}=2 \sqrt{\frac{-\lambda}{24}}, \lambda_{2}=\lambda_{3}=\sqrt{\frac{-\lambda}{24}}$ and $\lambda_{4}=-4 \sqrt{\frac{-\lambda}{24}}$.

Now we put

$$
u=-\frac{1}{2} e_{1}+\frac{\varepsilon \sqrt{3}}{2} e_{4}, \quad \varepsilon= \pm 1
$$

Then we notice that

$$
f(u)=h\left(K_{u} u, u\right)=-\frac{1}{8} \lambda_{1}-\frac{9}{8} \lambda_{4}+\frac{3 \sqrt{3}}{8} \varepsilon f\left(e_{4}\right) .
$$

If we choose $\varepsilon$ such that $\varepsilon f\left(e_{4}\right)$ is positive, then

$$
f(u) \geq-\frac{1}{8} \lambda_{1}+\frac{18}{8} \lambda_{1}=\frac{17}{8} \lambda_{1}>\lambda_{1}
$$

which contradicts the maximality of $\lambda_{1}$. Thus this case cannot occur.

Expressing the equation $\hat{R} \cdot K=0$, using the expression for $K$ obtained in cases $\mathrm{A}$ and $\mathrm{B}$, we obtain the following system of equations.

Case A.
(A1) $4 a^{2} b-12 a b^{2}+8 b^{3}-20 a c^{2}+8 b c^{2}-5 \lambda a+10 \lambda b=0$,
(A2) $c\left(12 a^{2}+4 a b+4 b^{2}+4 c^{2}+5 \lambda\right)=0$,
(A3) $d\left(16 a b+20 b^{2}+28 c^{2}+24 d^{2}+15 \lambda\right)=0$,
(A4) $b\left(4 b^{2}+4 c^{2}-4 a b+5 \lambda\right)=0$,
(A5) $(a+2 b)\left(4 a b+4 b^{2}+4 c^{2}+12 d^{2}+5 \lambda\right)=0$,
(A6) $d\left(12 a^{2}+28 a b+20 b^{2}+4 c^{2}+5 \lambda\right)=0$,
(A7) $a b c=0$; 
Case B.

(B1) $b=0$,

(B2) $c=0$,

(B3) $a f=0$,

(B4) $3 a^{2}+\frac{10 \lambda}{7}=0$,

(B5) $2 a^{2}-a d+\frac{5}{7} \lambda=0$.

Now we will solve these systems explicitly.

Solving the equations in Case A. We already noted that if $a=0$ then $b=c$ $=d=0$. This is a solution of the system.

We call it solution (S1).

Suppose $a \neq 0$, then from (A7) it follows that $b=0$ or $c=0$. So we can consider the following cases.

(a) $b=c=0$. This is not possible since (A1) then implies that $\lambda a=0$.

(b) $b=0, c \neq 0$. Then (A1) implies that $c^{2}=-\frac{\lambda}{4}$ and (A2) implies that $a^{2}=\frac{-\lambda}{3}$. Moreover, (A5) gives $d^{2}=\frac{-\lambda}{3}$. All the other equations are satisfied. Setting $u=-\frac{1}{\sqrt{5}}\left(e_{2}+e_{3}-\sqrt{3} e_{4}\right)$, we notice that

$$
h\left(K_{u} u, u\right)=\sqrt{\frac{-5 \lambda}{3}}>\sqrt{\frac{-\lambda}{3}}=a,
$$

but this contradicts the fact that $f_{2}$ attains an absolute maximum at $e_{2}$.

(c) $b \neq 0, c=0, d=0$. The equation (A4) implies that $4 a b=4 b^{2}+5 \lambda$. Substituting this in (A5) we obtain that $a+2 b=0$, implying that $b<0$. Using (A4) we get $b^{2}=-\frac{5 \lambda}{12}$. We can compute easily that all the the other equations are satisfied. We will call this solution of the system (S2).

(d) $b \neq 0, c=0, d \neq 0$. Again (A4) implies that $4 a b=4 b^{2}+5 \lambda$. Substi. tuting this into (A6) we get that $(a+2 b)(3 a+2 b)=0$.

If $a=-2 b$, then $b=-\sqrt{\frac{-5 \lambda}{12}}$. Substituting this into (A3) gives us $d=\sqrt{\frac{-5 \lambda}{6}}$. All the other equations are satisfied. We call this solution (S3). 
If $3 a+2 b=0$, then $b^{2}=-\frac{3 \lambda}{4}$. From (A5) we then obtain that $d=\frac{\sqrt{-3 \lambda}}{3}$ Setting $u=\frac{1}{\sqrt{2}}\left(-e_{2}+e_{3}\right)$ we get

$$
h\left(K_{u} u, u\right)=\frac{3}{4} \sqrt{-\frac{3 \lambda}{2}}>\sqrt{\frac{-\lambda}{3}},
$$

which again contradicts the fact that $f_{2}$ attains an absolute maximum at $e_{2}$.

Solving the equations in Case B. From the equation (B4) we conclude that $a=\sqrt{-\frac{10}{21} \lambda} \neq 0$. Thus (B3) implies that $f=0$. From (B5) we get $d=$ $\frac{1}{2} \sqrt{-\frac{10 \lambda}{21}}$ and all the other equations are satisfied.

If $u=-\cos \alpha e_{3}-\sin \alpha e_{4}, \alpha \in \mathbf{R}$, such that $\tan \alpha=\sqrt{\frac{7}{3}}$ then

$$
h\left(K_{u} u, u\right)=3 \sqrt{3} \sqrt{\frac{-\lambda}{21}}>\lambda_{1}
$$

which contradicts the fact that $\lambda_{1}$ is an absolute maximum.

The three possible shapes for $K$. Corresponding to the three possible solutions of the system (A), the following shapes for $K$ can occur at $p$.

(S1)

$$
\begin{aligned}
& K_{e_{1}} e_{1}=\frac{3 \sqrt{-\lambda}}{2} e_{1}, \\
& K_{e_{2}} e_{2}=K_{e_{3}} e_{3}=K_{e_{4}} e_{4}=-\frac{\sqrt{-\lambda}}{2} e_{1}, \\
& K_{e_{1}} e_{2}=-\frac{\sqrt{-\lambda}}{2} e_{2}, K_{e_{1}} e_{3}=\frac{\sqrt{-\lambda}}{2} e_{3}, K_{e_{1}} e_{4}=-\frac{\sqrt{-\lambda}}{2} e_{4}, \\
& K_{e_{2}} e_{3}=K_{e_{2}} e_{4}=K_{e_{3}} e_{4}=0 ;
\end{aligned}
$$

$$
\begin{aligned}
& K_{e_{1}} e_{1}=\frac{3 \sqrt{-\lambda}}{2} e_{1}, \\
& K_{e_{2}} e_{2}=\frac{-\sqrt{-\lambda}}{2} e_{1}+2 \sqrt{\frac{-5 \lambda}{12}} e_{2},
\end{aligned}
$$




$$
\begin{aligned}
& K_{e_{3}} e_{3}=-\frac{\sqrt{-\lambda}}{2} e_{1}-\sqrt{\frac{-5 \lambda}{12}} e_{2}, \\
& K_{e_{4}} e_{4}=-\frac{\sqrt{-\lambda}}{2} e_{1}-\sqrt{\frac{-5 \lambda}{12}} e_{2}, \\
& K_{e_{1}} e_{2}=-\frac{\sqrt{-\lambda}}{2} e_{2}, K_{e_{1}} e_{3}=-\frac{\sqrt{-\lambda}}{2} e_{3}, K_{e_{1}} e_{4}=-\frac{\sqrt{-\lambda}}{2} e_{4}, \\
& K_{e_{2} e_{3}}=-\sqrt{-\frac{5 \lambda}{12}} e_{3}, K_{e_{2}} e_{4}=-\sqrt{-\frac{5 \lambda}{12}} e_{4}, K_{e_{3} e_{4}}=0 ;
\end{aligned}
$$

$$
\begin{aligned}
& K_{e_{1}} e_{1}=\frac{3 \sqrt{-\lambda}}{2} e_{1}, \\
& K_{e_{2}} e_{2}=-\frac{\sqrt{-\lambda}}{2} e_{1}+2 \sqrt{-\frac{5 \lambda}{21}} e_{2}, \\
& K_{e_{3}} e_{3}=-\frac{\sqrt{-\lambda}}{2} e_{1}-\sqrt{\frac{-5 \lambda}{12}} e_{2}+\sqrt{\frac{-5 \lambda}{6}} e_{3}, \\
& K_{e_{4}} e_{4}=-\frac{\sqrt{-\lambda}}{2} e_{1}-\sqrt{\frac{-5 \lambda}{12}} e_{2}-\sqrt{\frac{-5 \lambda}{6}} e_{3}, \\
& K_{e_{1}} e_{2}=-\frac{\sqrt{-\lambda}}{2} e_{2}, K_{e_{1}} e_{3}=-\frac{\sqrt{-\lambda}}{2} e_{3}, K_{e_{1}} e_{4}=-\frac{\sqrt{-\lambda}}{2} e_{4}, \\
& K_{e_{2}} e_{3}=-\sqrt{\frac{-5 \lambda}{12}} e_{3}, K_{e_{2}} e_{4}=-\sqrt{\frac{-5 \lambda}{12}} e_{4}, K_{e_{3}} e_{4}=\sqrt{\frac{-5 \lambda}{12}} e_{4} .
\end{aligned}
$$

The following lemma can be proved as [DV1, Lemma 3.4].

LEMMA 3.1. If the case (S3) holds at $p$ then all the sectional curvatures are zero, moreover $h(K, K)=-\frac{67}{12} \lambda$. If the case $(\mathrm{S} 1)$ holds at $p$ then $h(K, K)=-\frac{9}{2} \lambda$ and if the case $(\mathrm{S} 2)$ holds at $p$ then $h(K, K)=-\frac{26}{3} \lambda$.

We therefore can conclude that, if (S1), respectively (S2) or (S3), is true at a point $p$, then it is true for every point on $M$. If (S3) is true on $M$, then we can apply the main theorem of [VLS] and obtain that $M$ is affine equivalent to an open part of the hypersurface (1) of Theorem 1.

Having this basis $\left\{e_{\imath}\right\}$ at a point $p$, we can translate it parallelly along geodesics through $p$ and obtain a local frame $\left\{E_{i}\right\}$ on a normal neighborhood of $p$. Since $\hat{\nabla} K=0, K$ will have the same expression in any point as in $p$. This is stated in the following lemmas, which can be proved similarly as Lemma 3.5 and Lemma 3.6 of [DV1]. 
LEMMA 3.2. Let $M$ be a 4-dimensional locally strongly convex affine hypersurface in $\mathbf{R}^{5}$ with $\hat{\nabla} C=0$. If (S1) holds at every point of $M$, then there exists a local basis $\left\{E_{1}, E_{2}, E_{3}, E_{4}\right\}$, orthonormal with respect to $h$, such that:

(1) at any $p \in M, f$ attains its maximum value at $E_{1}(p)$,

(2) at any $p \in M,\left\{E_{1}(p), E_{2}(p), E_{3}(p), E_{4}(p)\right\}$ satisfies (S1)

(3) $\hat{\nabla}_{X} E_{1}=0$, for any vector field $X$ on $M$.

Moreover, $(M, h)$, considered as a Riemannian manifold, is locally isometric to $\mathbf{R} \times H$, where $H$ is the 3-dimensional hyperbolic space of constant negative sectional curvature $\frac{5 \lambda}{4}$. After the identification, $E_{1}$ is tangent to $\mathbf{R}$.

LEMma 3.3. Let $M$ be a 4-dimensional locally strongly convex affine hypersurface in $\mathbf{R}^{5}$ with $\hat{\nabla} C=0$. If (S2) holds at every point of $M$, then there exists a local basis $\left\{E_{1}, E_{2}, E_{3}, E_{4}\right\}$ orthonormal with respect to $h$, such that:

(1) at any $p \in M, f$ attains its maximum value at $E_{1}(p)$,

(2) at any $p \in M,\left\{E_{1}(p), E_{2}(p), E_{3}(p), E_{4}(p)\right\}$ satisfies (S2),

(3) $\hat{\nabla}_{X} E_{1}=\hat{\nabla}_{X} E_{2}=0$, for any vector field $X$ on $M$.

Moreover, $(M, h)$, considered as a Riemannian manifold is isometric to $\mathbf{R} \times \mathbf{R} \times H$, where $H$ is the hyperbolic plane of constant negative sectional curvature $\frac{5 \lambda}{3}$. After the identification, $E_{1}$ is tangent to the first $\mathbf{R}$-component and $E_{2}$ is tangent to the second.

\section{§4. Proof of Theorem 1}

Using [DV2], it is easy to compute that the hypersurface (2) of Theorem 1 satisfies the data of Lemma 3.2, and that the hypersurface (3) satisfies Lemma 3.3 for some appropriate choice of $\lambda$.

Let $M$ satisfy Lemma 3.2, and suppose that $F: M \rightarrow \mathbf{R} \times H$ is an isometry (we should rather consider a suitable open subset of $M$, but we don't really worry about this). Let $f: \mathbf{R} \times H \rightarrow \mathbf{R}^{n+1}$ be the immersion giving the hypersurface (2), where we apply a homothetic transformation to make sure that both scaling factors $\lambda$ are the same, and let $g: M \rightarrow \mathbf{R}^{n+1}$ denote the immersion of $M$.

Let $\left\{E_{1}, E_{2}, E_{3}, E_{4}\right\}$ be the frame on $M$ satisfying Lemma 3.2 . Then it can be seen easily that $\left\{F_{*} E_{1}, F_{*} E_{2}, F_{*} E_{3}, F_{*} E_{4}\right\}$ is a frame on $\mathbf{R} \times H$ such that the difference tensor of $\mathbf{R} \times H$ has the form (S1). Hence $F$ preserves both the affine metric $h$ and the cubic form $C$. Applying the fundamental uniqueness theorem of affine differential geometry, for instance [D, Theorem 3.5], we obtain that there is an affine transformation $A: \mathbf{R}^{n+1} \rightarrow \mathbf{R}^{n+1}$ such that $A(g)=f(F)$. This means, 
forgetting about the immersions, that $M$ is affine equivalent to an open part of (2).

If $M$ satisfies Lemma 3.3, we can show similarly that it is affine equivalent to an open part of (3).

\section{§5. Proof of Theorem 2}

The fact that $M$ is an affine sphere follows from [BNS]. If $M$ satisfies $\hat{\nabla} C=0$, then $\hat{\nabla} \hat{R}=0$. Let $p, q \in M$ and let $\left\{e_{i}\right\}$ be any orthonormal basis of $T_{p} M$. We can translate it parallelly along geodesics through $p$ and obtain a local frame $\left\{E_{i}\right\}$ on a normal neighborhood of $p$. Since $\hat{\nabla} K=0$ and $\hat{\nabla} \hat{R}=0$, the numbers $c_{i j k}$ $=h\left(K\left(E_{i}, E_{j}\right), E_{k}\right)$ and $r_{i j k l}=h\left(\hat{R}\left(E_{i}, E_{j}\right) E_{k}, E_{l}\right)$ will be constants. If we translate $\left\{e_{i}\right\}$ parallelly to $q$, we obtain an orthonormal basis $\left\{f_{\imath}\right\}$ of $T_{q} M$. Let $L$ : $T_{p} M \rightarrow T_{q} M$ be the linear isometry mapping $e_{i}$ onto $f_{i}$. Then $L$ preserves curvature, such that from [O'N, Theorem 8.14] we know that there is an isometry $f: U$ $\rightarrow M$ from an open $U$ around $p$ such that $f(p)=q$ and $f_{* p}=L$. Let $F_{i}=f_{*} E_{i}$, then the frame $\left\{F_{i}\right\}$ is obtained from $\left\{f_{i}\right\}$ by parallel translation as above. Moreover $c_{i j k}=h\left(K\left(F_{i}, F_{j}\right), F_{k}\right)$ and $r_{i j k l}=h\left(\hat{R}\left(F_{i}, F_{j}\right) F_{k}, F_{l}\right)$ are the same constants. Therefore $f$ preserves both $h$ and $K$, so by the fundamental uniqueness theorem, we again obtain that there is an equiaffine transformation $A$ of $\mathbf{R}^{n+1}$ such that $A(x)=f(x)$ for all $x \in U$. Hence $M$ is locally homogeneous.

\section{REFERENCES}

[D] F. Dillen, Equivalence theorems in affine differential geometry, Geom. Dedicata, 32 (1989), 81-92.

[DV1] F. Dillen, L. Vrancken, 3-dimensional affine hypersurfaces in $\mathbf{R}^{4}$ with parallel cubic form, Nagoya Math, J., 124 (1991), 41-53.

[DV2] - Calabi-type composition of affine spheres, Diff. Geom. and Appl. (to appear).

[DV3] - The classification of 3-dimensional homogeneous locally strongly convex affine hypersurfaces, Manuscripta Math., 80 (1993), 165-180.

[MN] M. Magid and K. Nomizu, On affine surfaces whose cubic forms are parallel relative to the affine metric, Proc. Japan. Acad. Ser. A, 65 (1989), 215-218.

[N] K. Nomizu, Introduction to affine differential geometry, Part I, MPI / 88-38, Bonn, 1988, Revised: Department of Mathematics, Brown University, 1989.

[NS] K. Nomizu, T. Sasaki, A new model of unimodular-affinely homogeneous surfaces, Manuscripta Math., 73 (1991), 39-44.

[O'N] B. O'Neill, Semi-Riemannian geometry with applications to relativity, Academic Press, New York, 1983.

[VLS] L. Vrancken, A.-M. Li, U. Simon, Affine spheres with constant sectional curvature, Math. Z., 206 (1991), 651-658. 
$1,2,3$

Katholieke Universiteit Leuven

Departement Wiskunde

Celestijnenlaan $200 B$

B-3001 Leuven

Belgium

3

The University of Ankara

The Faculty of Sciences

Tandogan 06100

Ankara

Turkey 